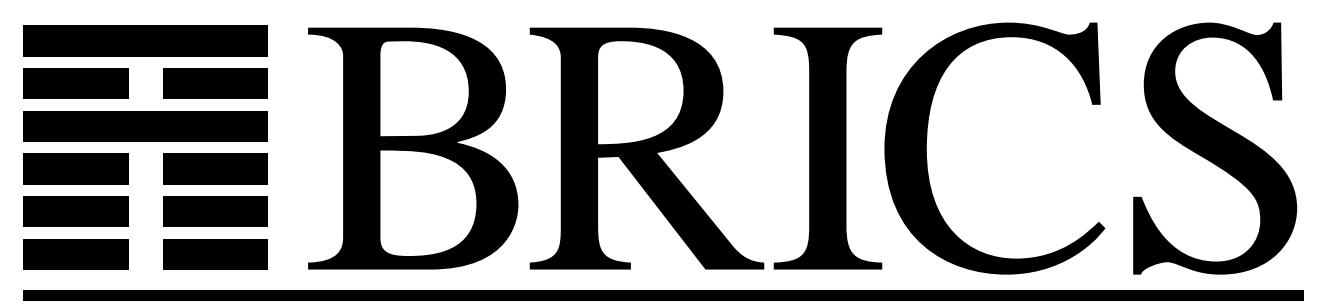

Basic Research in Computer Science

\title{
A Note on an Expressiveness Hierarchy for Multi-exit Iteration
}

Luca Aceto

Willem Jan Fokkink

Anna Ingólfsdóttir

RS-02-40 
Copyright (c) 2002, Luca Aceto \& Willem Jan Fokkink \& Anna Ingólfsdóttir.

BRICS, Department of Computer Science

University of Aarhus. All rights reserved.

Reproduction of all or part of this work is permitted for educational or research use on condition that this copyright notice is included in any copy.

See back inner page for a list of recent BRICS Report Series publications. Copies may be obtained by contacting:

\author{
BRICS \\ Department of Computer Science \\ University of Aarhus \\ Ny Munkegade, building 540 \\ DK-8000 Aarhus C \\ Denmark \\ Telephone: +4589423360 \\ Telefax: $\quad+4589423255$ \\ Internet: BRICS@brics.dk
}

BRICS publications are in general accessible through the World Wide Web and anonymous FTP through these URLs:

http: //www.brics.dk

ftp://ftp.brics.dk

This document in subdirectory RS / 02 / 40 / 


\title{
A Note on an Expressiveness Hierarchy for Multi-exit Iteration
}

\author{
Luca Aceto* Wan Fokkink ${ }^{\dagger} \quad$ Anna Ingólfsdóttir*‡
}

\begin{abstract}
Multi-exit iteration is a generalization of the standard binary Kleene star operation that allows for the specification of agents that, up to bisimulation equivalence, are solutions of systems of recursion equations of the form

$$
\begin{array}{ccc}
X_{1} & \stackrel{\text { def }}{=} & P_{1} X_{2}+Q_{1} \\
& \vdots & \\
X_{n} & \stackrel{\text { def }}{=} & P_{n} X_{1}+Q_{n}
\end{array}
$$

where $n$ is a positive integer, and the $P_{i}$ and the $Q_{i}$ are process terms. The addition of multi-exit iteration to Basic Process Algebra (BPA) yields a more expressive language than that obtained by augmenting BPA with the standard binary Kleene star. This note offers an expressiveness hierarchy, modulo bisimulation equivalence, for the family of multi-exit iteration operators proposed by Bergstra, Bethke and Ponse.
\end{abstract}

AMS Subject Classification (1991): 68Q15, 68Q70.

CR Subject Classification (1991): D.3.1, F.1.1, F.4.1.

Keywords And Phrases: Concurrency, process algebra, Basic Process Algebra (BPA), multi-exit iteration, bisimulation, expressiveness.

\section{Background}

For the sake of completeness and readability, we begin by recalling the relevant notions from 1 that will be needed in this note. The interested reader is referred to op. cit. and [5] for motivation and further information.

We assume a non-empty alphabet $A$ of atomic actions, with typical elements $a, b$. The language $\mathrm{BPA}^{m e *}(A)$ of terms over Basic Process Algebra (BPA) with multi-exit iteration is defined inductively as follows:

*BRICS (Basic Research in Computer Science), Centre of the Danish National Research Foundation, Department of Computer Science, Aalborg University, Fr. Bajersvej 7E, 9220 Aalborg $\varnothing$, Denmark. Email: \{luca, annai\}@cs.auc.dk.

${ }^{\dagger}$ CWI, Department of Software Engineering, Kruislaan 413, 1098 SJ Amsterdam, The Netherlands. Email: wan@cwi.nl.

${ }^{\ddagger}$ deCODE Genetics, Sturlugata 8, 101 Reykjavik, Iceland. 
- each $a \in A$ is a term;

- $P+Q$ and $P \cdot Q$ are terms, if so are $P$ and $Q$;

- $\left(P_{1}, \ldots, P_{m}\right)^{*}\left(Q_{1}, \ldots, Q_{n}\right)$ is a term, if so are $P_{1}, \ldots, P_{m}$ and $Q_{1}, \ldots, Q_{n}$ for some positive integers $m$ and $n$.

We shall use $P, Q, R$ (possibly subscripted and/or superscripted) to range over $\operatorname{BPA}^{m e *}(A)$. In writing terms over the above syntax, we shall always assume that the operation - binds stronger than + . In the sequel the operation - will often be omitted, so $P Q$ denotes $P \cdot Q$. We shall use the symbol $\equiv$ to stand for syntactic equality of terms. For every natural number $n$, we shall write $[n]$ in lieu of $\{1, \ldots, n\}$.

Apart from actions, the signature of the language $\operatorname{BPA}^{m e *}(A)$ includes the binary operations of alternative composition + and sequential composition . familiar from the theory of Basic Process Algebra [6, 4], and a variation on the original binary version of the Kleene star operation [9], that will be referred to as multi-exit iteration. For positive integers $m$ and $n$, the process term $\left(P_{1}, \ldots, P_{m}\right)^{*}\left(Q_{1}, \ldots, Q_{n}\right)$ stands for an agent whose behaviour is specified by the following defining equation:

$$
\left(P_{1}, \ldots, P_{m}\right)^{*}\left(Q_{1}, \ldots, Q_{n}\right)=P_{1} \cdot\left(P_{2}, \ldots, P_{m}, P_{1}\right)^{*}\left(Q_{2}, \ldots, Q_{n}, Q_{1}\right)+Q_{1} .
$$

In order to simplify notation in the presentation of the operational semantics for $\mathrm{BPA}^{m e *}(A)$, we shall use the notion of 'vectors of processes'. A vector of processes is a tuple $\left(P_{1}, \ldots, P_{m}\right)$, where $m \geq 0$. We shall use $\vec{Q}, \vec{S}$ to denote such vectors of processes. In multi-exit iteration, the expressions at the leftand right-hand sides of the star are non-empty vectors of processes. Enclosing parentheses will always be omitted from vectors of length one, i.e., $(P)$ will be written $P$.

The operational semantics for the language $\operatorname{BPA}^{m e *}(A)$ is given by the labelled transition system

$$
\left(\operatorname{BPA}^{m e *}(A),\{\stackrel{a}{\rightarrow} \mid a \in A\},\{\stackrel{a}{\rightarrow} \checkmark \mid a \in A\}\right)
$$

where the transition relations $\stackrel{a}{\rightarrow}$ and the unary predicates $\stackrel{a}{\rightarrow} \checkmark$ are, respectively, the least subsets of $\operatorname{BPA}^{m e *}(A) \times \operatorname{BPA}^{m e *}(A)$ and $\operatorname{BPA}^{m e *}(A)$ satisfying the rules in Table 1. Intuitively, a transition $P \stackrel{a}{\rightarrow} Q$ means that the system represented by the term $P$ can perform the action $a$, thereby evolving into $Q$. The special symbol $\checkmark$ stands for (successful) termination; therefore the interpretation of the statement $P \stackrel{a}{\rightarrow} \checkmark$ is that the process term $P$ can terminate by performing $a$. Note that, for every term $P$, there is some action $a$ for which either $P \stackrel{a}{\rightarrow} P^{\prime}$ holds for some $P^{\prime}$, or $P \stackrel{a}{\rightarrow} \checkmark$ does.

Definition 1.1 The term $P^{\prime}$ is a derivative of $P$ if $P$ can evolve into $P^{\prime}$ by zero or more transitions. $A$ derivative $P^{\prime}$ of $P$ is proper if $P$ can evolve into $P^{\prime}$ by performing at least one transition. 


$$
\begin{aligned}
& a \stackrel{a}{\rightarrow} \checkmark \\
& \frac{\stackrel{\stackrel{a}{\rightarrow} \checkmark}{P}}{P+Q \stackrel{a}{\rightarrow} \checkmark} \quad \frac{Q \stackrel{a}{\rightarrow} \checkmark}{P+Q \stackrel{a}{\rightarrow} \checkmark} \quad \frac{P \stackrel{a}{\rightarrow} P^{\prime}}{P+Q \stackrel{a}{\rightarrow} P^{\prime}} \quad \frac{Q \stackrel{a}{\rightarrow} Q^{\prime}}{P+Q \stackrel{a}{\rightarrow} Q^{\prime}} \\
& \frac{P \stackrel{a}{\rightarrow} \checkmark}{P \cdot Q \stackrel{a}{\rightarrow} Q} \quad \frac{P \stackrel{a}{\rightarrow} P^{\prime}}{P \cdot Q \stackrel{a}{\rightarrow} P^{\prime} \cdot Q} \\
& \frac{P \stackrel{a}{\rightarrow} \checkmark}{(P, \vec{Q})^{*}(R, \vec{S}) \stackrel{a}{\rightarrow}(\vec{Q}, P)^{*}(\vec{S}, R)} \quad \frac{P \stackrel{a}{\rightarrow} P^{\prime}}{(P, \vec{Q})^{*}(R, \vec{S}) \stackrel{a}{\rightarrow} P^{\prime} \cdot(\vec{Q}, P)^{*}(\vec{S}, R)} \\
& \frac{R \stackrel{a}{\rightarrow} \checkmark}{(P, \vec{Q})^{*}(R, \vec{S}) \stackrel{a}{\rightarrow} \checkmark} \quad \frac{R \stackrel{a}{\rightarrow} R^{\prime}}{(P, \vec{Q})^{*}(R, \vec{S}) \stackrel{a}{\rightarrow} R^{\prime}}
\end{aligned}
$$

Table 1: Transition Rules

Process terms are considered modulo bisimulation equivalence [10].

Definition 1.2 Two process terms $P$ and $Q$ are bisimilar, denoted by $P \leftrightarrows Q$, if there exists a symmetric binary relation $\mathcal{B}$ on process terms which relates $P$ and $Q$, such that:

- if $R \mathcal{B} S$ and $R \stackrel{a}{\rightarrow} R^{\prime}$, then there is a transition $S \stackrel{a}{\rightarrow} S^{\prime}$ such that $R^{\prime} \mathcal{B} S^{\prime}$,

- if $R \mathcal{B} S$ and $R \stackrel{a}{\rightarrow}$, then $S \stackrel{a}{\rightarrow} \checkmark$.

Such a relation $\mathcal{B}$ will be called a bisimulation. The relation $\leftrightarrows$ will be referred to as bisimulation equivalence.

Note that if $P$ is bisimilar to $Q$, then every (proper) derivative of $P$ is bisimilar to some (proper) derivative of $Q$, and vice versa.

The transition rules in Table 1 are in the 'path' format of Baeten and Verhoef [3]. Hence, bisimulation equivalence is a congruence with respect to all the operations in the signature of $\mathrm{BPA}^{\text {me* }}(A)$.

Process terms in $\mathrm{BPA}^{m e *}(A)$ are normed, which means that they are able to terminate by embarking in a finite sequence of transitions. We call such a sequence a termination trace. The norm of a process term $P$, denoted by $|P|$, is the length of its shortest termination trace; this notion stems from [2. Note that bisimilar process terms have the same norm. The following lemma, which is due to Caucal [8], is typical for normed processes, and will be useful in the technical developments to follow.

Lemma 1.3 Let $P, Q, R, S \in \mathrm{BPA}^{m e *}(A)$ be such that $P Q \leftrightarrows R S$. If $|Q|=|S|$, then $P \leftrightarrows R$ and $Q \leftrightarrows S$.

A technical tool we shall use below is a weight function $g$ that associates a 
natural number to each process term. This is defined thus:

$$
\begin{aligned}
g(a) & \triangleq 0 \\
g(P+Q) & \triangleq \max \{g(P), g(Q)\}+1 \\
g(P Q) & \triangleq \max \{g(P), g(Q)\} \\
g\left(\left(P_{1}, \ldots, P_{m}\right)^{*}\left(Q_{1}, \ldots, Q_{n}\right)\right) & \triangleq \max \left\{g\left(P_{i}\right), g\left(Q_{j}\right)+1 \mid i \in[m], j \in[n]\right\} .
\end{aligned}
$$

The basic property of this weight function that we shall need is expressed in the lemma below (cf. [1, Lemma 3.5]).

Lemma 1.4 If $P^{\prime}$ is a derivative of $P$, then $g\left(P^{\prime}\right) \leq g(P)$. Moreover, if

- $P \equiv P_{1}+P_{2}$ for some terms $P_{1}$ and $P_{2}$, and $P^{\prime}$ is a proper derivative of $P$, or

- $P \equiv\left(P_{1}, \ldots, P_{m}\right)^{*}\left(Q_{1}, \ldots, Q_{n}\right)$, for some terms $P_{i}(i \in[m])$ and $Q_{j}(j \in$ $[n])$, and $P^{\prime}$ is a proper derivative of some $Q_{j}$,

then $g\left(P^{\prime}\right)<g(P)$.

\section{An Expressiveness Hierarchy}

As shown in [5], the addition of multi-exit iteration to BPA yields a language that, modulo bisimulation equivalence, is strictly more expressive than that obtained by augmenting BPA with the standard binary Kleene star. More precisely, it is proven ibidem that, in the presence of at least two actions, the process $(a, a)^{*}(a, b)$ cannot be expressed, modulo bisimulation equivalence, in ACP 4 , and a fortiori in BPA, enriched with the binary Kleene star (cf. Lemma 3.2.3 in op. cit.).

Let us say that a term of the form $\left(P_{1}, \ldots, P_{m}\right)^{*}\left(Q_{1}, \ldots, Q_{n}\right)$ has $n$-exit iteration. By analogy with the aforementioned result from [5], it was proved in [1] that, in the presence of a non-empty set of actions, the sequence of $k$ exit iteration operations induces a hierarchy of super-languages of BPA with a strictly increasing expressive power modulo bisimulation equivalence. To this end, it was shown in op. cit. that, for every positive integer $k$, the process

$$
a^{*}\left(a, a^{2}, \ldots, a^{k+1}\right)
$$

cannot be specified using $h$-exit iteration with $h \leq k$, modulo bisimulation equivalence. (Cf. Corollary 4.5 in [1.)

In light of the above result, increasing the maximum number of exits allowed in a multi-exit iteration increases the expressive power of the language modulo bisimulation equivalence. Our aim in this note is to show that increasing the maximum number of processes on the left-hand side of the star in a multi-exit iteration also increases the expressive power of the language modulo bisimulation equivalence. 
Notation 1 For every positive integer $k$, we write $\mathrm{BPA}^{k *}$ for the set of terms in the language $\mathrm{BPA}^{\text {me* }}(A)$ that may use multi-exit iteration operations whose first argument is a non-empty vector of processes of length at most $k$.

For a positive integer $i$ and action $a$, we write $a^{i}$ for the term obtained by concatenating $i$ copies of action $a$.

Our aim is to prove the following theorem:

Theorem 2.1 For every positive integer $k$, the process $\left(a, a^{2}, \ldots, a^{k+1}\right)^{*} a$ cannot be expressed in the language $\mathrm{BPA}^{k *}$ modulo bisimulation equivalence.

The remainder of this note will be devoted to a proof of the above result. To this end, it is sufficient to establish the following special case of the statement of our main result.

Proposition 2.2 For every positive integer $k$, the process $\left(a, a^{2}, \ldots, a^{k+1}\right)^{*} a$ cannot be expressed, modulo bisimulation equivalence, as a term in the language $\mathrm{BPA}^{k *}$ of the form $\left(P_{1}, \ldots, P_{h}\right)^{*}\left(Q_{1}, \ldots, Q_{m}\right)$ with $\left|Q_{j}\right|=1$, for every $j \in[m]$.

Indeed, using the above result, we can prove Theorem 2.1 thus:

Proof of Theorem 2.1: Assume, towards a contradiction, that there is a term $P$ in the language $\mathrm{BPA}^{k *}$ that is bisimilar to $\left(a, a^{2}, \ldots, a^{k+1}\right)^{*} a$. Assume, furthermore, that $P$ is a process with this property with minimum weight $g(P)$. We proceed with the proof by analyzing the possible forms such a $P$ may take.

It is easy to see that $P$ can neither have the form $a$ nor the form $P_{1} P_{2}$ for some processes $P_{1}$ and $P_{2}$. Indeed, this follows because bisimilar processes have equal norm, but any process of the form $P_{1} P_{2}$ has norm at least two and $\left(a, a^{2}, \ldots, a^{k+1}\right)^{*} a$ has norm one.

We claim that $P$ cannot have the form $\left(P_{1}, \ldots, P_{h}\right)^{*}\left(Q_{1}, \ldots, Q_{m}\right)$ either. To see this, note, first of all, that, by Proposition 2.2, $P$ cannot have the form $\left(P_{1}, \ldots, P_{h}\right)^{*}\left(Q_{1}, \ldots, Q_{m}\right)$, with $\left|Q_{j}\right|=1$ for every $j \in[m]$. If there is some $Q_{j}(j \in[m])$ whose norm is greater than one, then this $Q_{j}$ affords a transition $Q_{j} \stackrel{a}{\rightarrow} Q_{j}^{\prime}$ for some process $Q_{j}^{\prime}$. It follows that, for some positive integer $\ell$,

$$
\left(P_{1}, \ldots, P_{h}\right)^{*}\left(Q_{1}, \ldots, Q_{m}\right) \stackrel{a^{\ell}}{\rightarrow} Q_{j}^{\prime}
$$

Since the terms $\left(P_{1}, \ldots, P_{h}\right)^{*}\left(Q_{1}, \ldots, Q_{m}\right)$ and $\left(a, a^{2}, \ldots, a^{k+1}\right)^{*} a$ are bisimilar, there is a derivative $R$ of the latter term such that

$$
\left(a, a^{2}, \ldots, a^{k+1}\right)^{*} a \stackrel{a^{\ell}}{\rightarrow} R \quad \text { and } \quad Q_{j}^{\prime} \leftrightarrows R
$$

As $\left(a, a^{2}, \ldots, a^{k+1}\right)^{*} a$ is easily seen to be a derivative of $R$, we have that $Q_{j}^{\prime}$ has a derivative that is bisimilar to $\left(a, a^{2}, \ldots, a^{k+1}\right)^{*} a$, and thus that $Q_{j}$ has a proper derivative $Q^{\prime}$ that is bisimilar to $\left(a, a^{2}, \ldots, a^{k+1}\right)^{*} a$. By Lemma 1.4 the value of $g\left(Q^{\prime}\right)$ is strictly smaller than $g(P)$. This contradicts our assumption that $P$ was a process with minimum weight in $\mathrm{BPA}^{k *}$ that is bisimilar to $\left(a, a^{2}, \ldots, a^{k+1}\right)^{*} a$. 
From the above reasoning, it follows that $P$ can only have the form $P_{1}+P_{2}$ for some processes $P_{1}$ and $P_{2}$. Since

$$
\left(a, a^{2}, \ldots, a^{k+1}\right)^{*} a \stackrel{a^{n}}{\rightarrow}\left(a, a^{2}, \ldots, a^{k+1}\right)^{*} a \quad\left(n=\frac{(k+1)(k+2)}{2}\right)
$$

and $P \equiv P_{1}+P_{2}$ is bisimilar to $\left(a, a^{2}, \ldots, a^{k+1}\right)^{*} a$, there is a process $P^{\prime}$ such that

$$
P \stackrel{a^{n}}{\rightarrow} P^{\prime} \quad \text { and } \quad P^{\prime} \leftrightarrows\left(a, a^{2}, \ldots, a^{k+1}\right)^{*} a
$$

By Lemma 1.4, since $P^{\prime}$ is a proper derivative of $P \equiv P_{1}+P_{2}$, the value of $g\left(P^{\prime}\right)$ is strictly smaller than $g(P)$. This contradicts our assumption that $P$ was a process with minimum weight in $\mathrm{BPA}^{k *}$ that is bisimilar to $\left(a, a^{2}, \ldots, a^{k+1}\right)^{*} a$.

It follows that no term in $\mathrm{BPA}^{k *}$ can be bisimilar to $\left(a, a^{2}, \ldots, a^{k+1}\right)^{*} a$, which was to be shown.

To complete the proof, we are therefore left to show Proposition 2.2, This result is an immediate consequence of the second statement in the following lemma.

Lemma 2.3 Assume that $Q_{1}, \ldots, Q_{m}$ are processes with norm one. Then the following statements hold:

1. For every positive integer $i$, if $\left(P_{1}, \ldots, P_{h}\right)^{*}\left(Q_{1}, \ldots, Q_{m}\right)$ is bisimilar to $\left(a^{i}, R_{1}, \ldots, R_{n}\right)^{*} a(n \geq 0)$, then

- $P_{1} \leftrightarrows a^{i}$ and

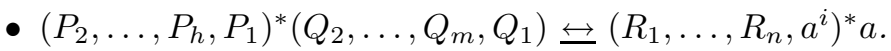

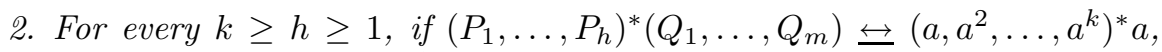
then $h=k$, and $P_{i} \leftrightarrows a^{i}$ for every $i \in[k]$.

Proof: We prove the two statements separately.

- Proof of Statement [1. We consider two cases, depending on whether $i=1$ or not. In both cases of the proof, we use the fact that, as

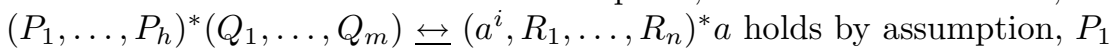
can perform an $a$-labelled transition and no transition labelled with actions different from $a$.

Assume that $i=1$. Then $P_{1}$ has no transitions of the form $P_{1} \stackrel{a}{\rightarrow} P_{1}^{\prime}$. Indeed, if $P_{1} \stackrel{a}{\rightarrow} P_{1}^{\prime}$ holds, then so does

$$
\left(P_{1}, \ldots, P_{h}\right)^{*}\left(Q_{1}, \ldots, Q_{m}\right) \stackrel{a}{\rightarrow} P_{1}^{\prime}\left(P_{2}, \ldots, P_{h}, P_{1}\right)^{*}\left(Q_{2}, \ldots, Q_{m}, Q_{1}\right) .
$$

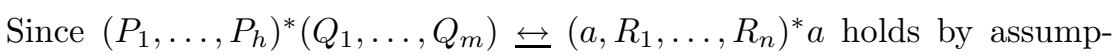
tion, there is a transition

$$
\left(a, R_{1}, \ldots, R_{n}\right)^{*} a \stackrel{a}{\rightarrow} R
$$

for some $R$ such that

$$
P_{1}^{\prime}\left(P_{2}, \ldots, P_{h}, P_{1}\right)^{*}\left(Q_{2}, \ldots, Q_{m}, Q_{1}\right) \leftrightarrows R
$$


The only candidate for this $R$ is the term $\left(R_{1}, \ldots, R_{n}, a\right)^{*} a$. However, the term $\left(R_{1}, \ldots, R_{n}, a\right)^{*} a$ has norm one, whereas

$$
\left|P_{1}^{\prime}\left(P_{2}, \ldots, P_{h}, P_{1}\right)^{*}\left(Q_{2}, \ldots, Q_{m}, Q_{1}\right)\right| \geq 2 .
$$

It follows that $P_{1}^{\prime}\left(P_{2}, \ldots, P_{h}, P_{1}\right)^{*}\left(Q_{2}, \ldots, Q_{m}, Q_{1}\right)$ cannot be bisimilar to $\left(R_{1}, \ldots, R_{n}, a\right)^{*} a$, and thus that $P_{1} \stackrel{a}{\rightarrow} \checkmark$ is the only transition afforded by $P_{1}$. We can now conclude that

$$
\begin{aligned}
& -P_{1} \leftrightarrows a \text { and } \\
& -\left(P_{2}, \ldots, P_{h}, P_{1}\right)^{*}\left(Q_{2}, \ldots, Q_{m}, Q_{1}\right) \leftrightarrows\left(R_{1}, \ldots, R_{n}, a\right)^{*} a
\end{aligned}
$$

both hold, which was to be shown.

Assume now that $i$ is greater than 1 . Reasoning as in the previous case, it is not hard to see that $P_{1}$ only affords transitions of the form $P_{1} \stackrel{a}{\rightarrow} P_{1}^{\prime}$. For every such transition, we have a transition of the form (1) out of $\left(P_{1}, \ldots, P_{h}\right)^{*}\left(Q_{1}, \ldots, Q_{m}\right)$. These transitions can only be matched by the transition

$$
\left(a^{i}, R_{1}, \ldots, R_{n}\right)^{*} a \stackrel{a}{\rightarrow} a^{i-1}\left(R_{1}, \ldots, R_{n}, a^{i}\right)^{*} a
$$

from $\left(a^{i}, R_{1}, \ldots, R_{n}\right)^{*} a$. It follows that, for every term $P_{1}^{\prime}$ such that $P_{1} \stackrel{a}{\rightarrow}$ $P_{1}^{\prime}$, it holds that

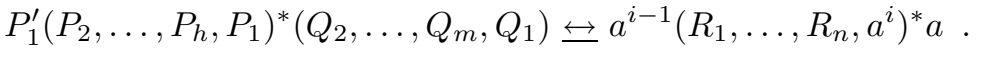

Since the terms $\left(P_{2}, \ldots, P_{h}, P_{1}\right)^{*}\left(Q_{2}, \ldots, Q_{m}, Q_{1}\right)$ and $\left(R_{1}, \ldots, R_{n}, a_{i}\right)^{*} a$ have both norm one by the proviso of the lemma, Lemma 1.3 yields that

$$
\begin{aligned}
& P_{1}^{\prime} \leftrightarrows a^{i-1} \quad \text { and }
\end{aligned}
$$

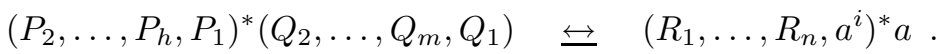

To complete the proof for this case, note that since every term that can be reached from $P_{1}$ via an $a$-labelled transition is bisimilar to $a^{i-1}$, from our previous observations it follows that $P_{1}$ is bisimilar to $a^{i}$.

- Proof of Statement 2 Assume that $k \geq h \geq 1$ and

$$
\left(P_{1}, \ldots, P_{h}\right)^{*}\left(Q_{1}, \ldots, Q_{m}\right) \leftrightarrows\left(a, a^{2}, \ldots, a^{k}\right)^{*} a
$$

Using statement 1 of the lemma repeatedly, we have that $P_{i} \leftrightarrows a^{i}$ for every $i \in[h]$, and

$$
\left(P_{1}, \ldots, P_{h}\right)^{*}\left(Q_{\ell+1}, \ldots, Q_{m}, Q_{1}, \ldots, Q_{\ell}\right) \leftrightarrows\left(a^{h+1}, \ldots, a^{k}, a_{1}, \ldots, a^{h}\right)^{*} a
$$

where $\ell=h \bmod m$.

If $h<k$, then statement 1 of the lemma would entail that

$$
a \leftrightarrows P_{1} \leftrightarrows a^{h+1}
$$

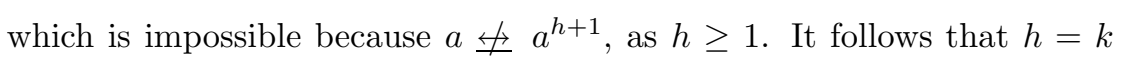
holds, and we are done. 
This completes the proof of the lemma.

Acknowledgements: The research reported in this note originated from a question posed to the authors by Kim G. Larsen.

\section{References}

[1] L. Aceto And W. J. Fokkink. An Equational Axiomatization for Multiexit Iteration. Information and Computation, 137(2):121-158, 15 September 1997.

[2] J. Baeten, J. Bergstra, And J. Klop, Decidability of bisimulation equivalence for processes generating context-free languages, J. Assoc. Comput. Mach., 40 (1993), pp. 653-682.

[3] J. BAEten AND C. Verhoef, A congruence theorem for structured operational semantics, in Best [7], pp. 477-492.

[4] J. Baeten and W. WeiJland, Process Algebra, Cambridge Tracts in Theoretical Computer Science 18, Cambridge University Press, 1990.

[5] J. Bergstra, I. Bethke, And A. Ponse, Process algebra with iteration, Report CS-R9314, Programming Research Group, University of Amsterdam, 1993.

[6] J. Bergstra AND J. KLOP, Fixed point semantics in process algebras, Report IW 206, Mathematisch Centrum, Amsterdam, 1982.

[7] E. Best, ed., Proceedings CONCUR 93, Hildesheim, Germany, vol. 715 of Lecture Notes in Computer Science, Springer-Verlag, 1993.

[8] D. CAucAl, Graphes canoniques de graphes algébriques, Theoretical Informatics and Applications, 24 (1990), pp. 339-352.

[9] S. KLEene, Representation of events in nerve nets and finite automata, in Automata Studies, C. Shannon and J. McCarthy, eds., Princeton University Press, 1956, pp. 3-41.

[10] D. PARK, Concurrency and automata on infinite sequences, in $5^{\text {th }}$ GI Conference, Karlsruhe, Germany, P. Deussen, ed., vol. 104 of Lecture Notes in Computer Science, Springer-Verlag, 1981, pp. 167-183. 


\section{Recent BRICS Report Series Publications}

RS-02-40 Luca Aceto, Willem Jan Fokkink, and Anna Ingólfsdóttir. A Note on an Expressiveness Hierarchy for Multi-exit Iteration. September 2002. 8 pp.

RS-02-39 Stephen L. Bloom and Zoltán Ésik. Some Remarks on Regular Words. September 2002.

RS-02-38 Daniele Varacca. The Powerdomain of Indexed Valuations. September 2002. 54 pp. Short version appears in Plotkin, editor, Seventeenth Annual IEEE Symposium on Logic in Computer Science, LICS '02 Proceedings, 2002, pages 299-308.

RS-02-37 Mads Sig Ager, Olivier Danvy, and Mayer Goldberg. A Symmetric Approach to Compilation and Decompilation. August 2002. To appear in Neil Jones's Festschrift.

RS-02-36 Daniel Damian and Olivier Danvy. CPS Transformation of Flow Information, Part II: Administrative Reductions. August 2002. 9 pp. To appear in the Journal of Functional Programming. This report supersedes the earlier BRICS report RS-0140.

RS-02-35 Patricia Bouyer. Timed Automata May Cause Some Troubles. August 2002. 44 pp.

RS-02-34 Morten Rhiger. A Foundation for Embedded Languages. August 2002. 29 pp.

RS-02-33 Vincent Balat and Olivier Danvy. Memoization in TypeDirected Partial Evaluation. July 2002. 18 pp. To appear in Batory and Consel, editors, ACM SIGPLAN/SIGSOFT Conference on Generative Programming and Component Engineering, GPCE' 02 Proceedings, LNCS, 2002.

RS-02-32 Mads Sig Ager, Olivier Danvy, and Henning Korsholm Rohde. On Obtaining Knuth, Morris, and Pratt's String Matcher by Partial Evaluation. July 2002. 43 pp. To appear in Chin, editor, ACM SIGPLAN ASIAN Symposium on Partial Evaluation and Semantics-Based Program Manipulation, ASIA-PEPM '02 Proceedings, 2002.

RS-02-31 Ulrich Kohlenbach and Paulo B. Oliva. Proof Mining: A Systematic Way of Analysing Proofs in Mathematics. June 2002. 47 pp. 